\title{
On subcritical multi-type branching process in random environment
}

\author{
Elena Dyakonova $\|^{\|}$ \\ ${ }^{1}$ Department of Discrete Mathematics, Steklov Mathematical Institute, Gubkin St. 8, 119991 Moscow, Russia
}

We investigate a multi-type Galton-Watson process in a random environment generated by a sequence of independent identically distributed random variables. Suppose that the associated random walk constructed by the logarithms of the Perron roots of the reproduction mean matrices has negative mean and assuming some additional conditions, we find the asymptotics of the survival probability at time $n$ as $n \rightarrow \infty$.

Keywords: branching processes in random environment, survival probability, limit theorems, random walks

\section{Introduction}

Branching processes in random environment are natural generalization of simple Galton-Watson processes. A branching process in random environment was first considered by Smith and Wilkinson (11). The subsequent papers investigated single- and multi-type Galton-Watson processes in random environment (3)-(8). A lot of papers is devoted to the study of the survival probability of single-type branching processes in random environment (see, for example, (1)-(9)). The asymptotics of the survival probability of the subcritical branching processes in random environment generated by a sequence of independent identically distributed random variables was found in (7) for single-type processes. The paper (5) deals with the asymptotics of the survival probability for the multi-type branching processes in random environment whose associated random walks satisfy the Doney-Spitzer condition.The present paper studies a case of subcritical multi-type processes branching processes in random environment. In particular, we generalize some results from (7) and (5).

Let $Z(n)=\left(Z_{1}(n), \ldots, Z_{p}(n)\right), n=0,1, \ldots$, be a $p$-type Galton-Watson branching process in a random environment. This process can be described as follows.

Let $\mathbf{N}_{0}=\{0,1,2, \ldots\}$ and $\mathbf{N}_{0}^{p}$ be the set of all vectors $t=\left(t_{1}, \ldots, t_{p}\right)$ with non-negative integer coordinates. Denote by $\left(\Delta_{1}, \mathcal{B}\left(\Delta_{1}\right)\right)$ a set of probability measures on $\mathbf{N}_{0}^{p}$ with $\sigma$-algebra $\mathcal{B}\left(\Delta_{1}\right)$ of Borel sets endowed with the metric of total variation, and by $(\Delta, \mathcal{B}(\Delta))$ the $p$-times product of the space $\left(\Delta_{1}, \mathcal{B}\left(\Delta_{1}\right)\right)$ on itself. Let $\mathbf{F}=\left(\mathbf{F}^{(1)}, \ldots, \mathbf{F}^{(p)}\right)$ be a random variable (random measure) taking values in $(\Delta, \mathcal{B}(\Delta))$. An infinite sequence $\Pi=\left(\mathbf{F}_{0}, \mathbf{F}_{1}, \mathbf{F}_{2}, \ldots\right)$ of independent identically distributed copies of $\mathbf{F}$ is said to form a random environment and we will say that $\mathbf{F}$ generates $\Pi$.

\footnotetext{
${ }^{\dagger}$ The work is supported by the Russian Science Foundation grant 08-01-00078 . 
A sequence of random $p$-dimensional vectors $Z(0), Z(1), Z(2), \ldots$ with non-negative integer coordinates is called a $p$-type branching process in random environment $\Pi$, if $Z(0)$ is independent of $\Pi$ and for all $n \geq 0, z=\left(z_{1}, \ldots, z_{p}\right) \in \mathbf{N}_{0}^{p}$ and $f_{0}, f_{1}, \ldots \in \Delta$

$$
\begin{aligned}
\mathcal{L}(Z & \left.(n+1) \mid Z(n)=\left(z_{1}, \ldots, z_{p}\right), \Pi=\left(f_{1}, f_{2}, \ldots\right)\right) \\
& =\mathcal{L}\left(Z(n+1) \mid Z(n)=\left(z_{1}, \ldots, z_{p}\right), \mathbf{F}_{n}=f_{n}\right) \\
& =\mathcal{L}\left(\sum_{i=1}^{p} \xi_{n, 1}^{(i)}+\ldots+\xi_{n, z_{i}}^{(i)}\right),
\end{aligned}
$$

where $f_{n}=\left(f_{n}^{(1)}, f_{n}^{(2)}, \ldots, f_{n}^{(p)}\right) \in \Delta, \xi_{n, 1}^{(i)}, \xi_{n, 2}^{(i)}, \ldots, \xi_{n, z_{i}}^{(i)}, i=1, \ldots, p$, are independent $p$-dimensional random vectors, and for each $i=1, \ldots, p$ the random vectors $\xi_{n, 1}^{(i)}, \xi_{n, 2}^{(i)}, \ldots, \xi_{n, z_{i}}^{(i)}$ are identically distributed according to the measure $f_{n}^{(i)}$. Relation $(1)$ defines a branching Galton-Watson process $Z(n)$ in random environment which describes the evolution of a particle population $Z(n)=\left(Z_{1}(n), \ldots, Z_{p}(n)\right), n=$ $0,1, \ldots$, where $Z_{i}(n), i=1, \ldots, p$, is the number of type $i$ particles in the $n$-th generation.

This population evolves as follows. If $\mathbf{F}_{n}=\left(f_{n}^{(1)}, \ldots, f_{n}^{(p)}\right) \in \Delta$ then each of the $Z_{i}(n)$ particles of type $i$ existing at the time $n$, produces offspring in accordance with the $p$-dimensional probability measure $f_{n}^{(i)}$ independently of the reproduction of other particles. Thus, the $i$-th component of the vector $Z(n+1)=\left(Z_{1}(n+1), \ldots, Z_{p}(n+1)\right)$ is equal to the number of type $i$ particles among all direct descendants of the particles of the $n$-th generation.

\section{The main results}

Let $J^{p}$ be the set of all column vectors $s=\left(s_{1}, \ldots, s_{p}\right)^{T}, 0 \leq s_{i} \leq 1, i=1, \ldots, p$. Here and later on the superscript $T$ stands for transposition. For $s=\left(s_{1}, \ldots, s_{p}\right)^{T}$ and $t=\left(t_{1}, \ldots, t_{p}\right) \in \mathbf{N}_{0}^{p}$ set $s^{t}=\prod_{i=1}^{p} s_{i}^{t_{i}}$. Taking into account existence of a one-to-one correspondence between probability measures and generating functions we associate with $\mathbf{F}=\left(\mathbf{F}^{(1)}, \ldots, \mathbf{F}^{(p)}\right)$ generating $\Pi$ a random $p$-dimensional column vector $F(s)=\left(F^{(1)}(s), \ldots, F^{(p)}(s)\right)^{T}, s \in J^{p}$, whose components are $p$-dimensional (random) generating functions $F^{(i)}(s)$ corresponding to $\mathbf{F}^{(i)}, 1 \leq i \leq p$ :

$$
F^{(i)}(s)=\sum_{t \in \mathbf{N}_{0}^{p}} \mathbf{F}^{(i)}(t) s^{t}, s \in J^{p}
$$

In a similar way we associate with the component $\mathbf{F}_{n}=\left(\mathbf{F}_{n}^{(1)}, \ldots, \mathbf{F}_{n}^{(p)}\right), n \geq 0$, of the random environment $\Pi=\left(\mathbf{F}_{0}, \mathbf{F}_{1}, \mathbf{F}_{2}, \ldots\right)$ a random vector $F_{n}(s)=\left(F_{n}^{(1)}(s), \ldots, F_{n}^{(p)}(s)\right)^{T}, s \in J^{p}$, the components of which are multidimensional (random) generating functions $F_{n}^{(i)}(s)$, corresponding to $\mathbf{F}_{n}^{(i)}, 1 \leq i \leq p$,

$$
F_{n}^{(i)}(s)=\sum_{t \in \mathbf{N}_{0}^{p}} \mathbf{F}_{n}^{(i)}(t) s^{t}
$$

Let $e_{j}, j=1, \ldots, p$, be the $p$-dimensional row vector whose $j$-th component is equal to 1 and the others are zeros, $\overline{0}=(0, \ldots, 0)$ be the $p$-dimensional row vector all whose components are zeros, and let $\overline{1}=$ $(1, \ldots, 1)^{T}$ be the $p$-dimensional column vector all whose components are equal to 1 . For $x=\left(x_{1}, \ldots, x_{p}\right)$ 
and $y=\left(y_{1}, \ldots, y_{p}\right)^{T}$ we set $|x|=\sum_{i=1}^{p}\left|x_{i}\right|,(x, y)=\sum_{i=1}^{p} x_{i} y_{i}$. Let $A=(A(i, j))_{i, j=1}^{p}$ be an arbitrary positive $p \times p$ matrix. Denote by $\rho(A)$ the Perron root of $A$ and by $u(A)=\left(u_{1}(A), \ldots, u_{p}(A)\right)^{T}$ and $v(A)=\left(v_{1}(A), \ldots, v_{p}(A)\right)$ the right and left eigenvectors of $A$ corresponding to the eigenvalue $\rho(A)$ and such that

$$
|v(A)|=1,(v(A), u(A))=1 .
$$

For vector-valued generating functions $F(s)$ and $F_{n}(s)$ we introduce the mean matrices

$$
M=M(\mathbf{F})=(M(i, j))_{i, j=1}^{p}=\left(\frac{\partial F^{(i)}(\overline{1})}{\partial s_{j}}\right)_{i, j=1}^{p}
$$

and

$$
M_{n}=M_{n}\left(\mathbf{F}_{n}\right)=\left(M_{n}(i, j)\right)_{i, j=1}^{p}=\left(\frac{\partial F_{n}^{(i)}(\overline{1})}{\partial s_{j}}\right)_{i, j=1}^{p} .
$$

Let $\mathcal{C}_{\alpha}, 0<\alpha<1$, be the class of all matrices $A=(A(i, j))_{i, j=1}^{p}$ such that

$$
\alpha \leq \frac{A\left(i_{1}, j_{1}\right)}{A\left(i_{2}, j_{2}\right)} \leq \alpha^{-1}, 1 \leq i_{1}, i_{2}, j_{1}, j_{2} \leq p .
$$

One of our basic hypotheses is the following condition.

Assumption A0. There exist a number $0<\alpha<1$ and a row vector $v=\left(v_{1}, \ldots, v_{p}\right), v_{i}>0, i=$ $1, \ldots, p,|v|=1$, such that

$$
M \in \mathcal{C}_{\alpha}
$$

and

$$
v(M)=v .
$$

Thus, all mean matrices $M_{n}, n \geq 0$, have a common left eigenvector $v$, i.e.,

$$
v M_{n}=\rho\left(M_{n}\right) v .
$$

Set $\rho=\rho(M), \rho_{n}=\rho\left(M_{n}\right), n \geq 0$. It is not difficult to see that in our settings $X:=\ln \rho, X_{i}:=$ $\ln \rho_{i-1}, i \geq 1$, are independent and identically distributed random variables. Our next hypothesis imposes a restriction on the so-called associated random walk $S=\left(S_{0}, S_{1}, \ldots\right)$, where

$$
S_{n}=X_{1}+\cdots+X_{n}, n \geq 1, S_{0}=0 .
$$

Assumption A1. Suppose that

$$
\mathbf{E}(\rho \log \rho)<0 .
$$

Note that, by means of Jensen inequality, Assumption A1 implies

$$
\mathbf{E} \rho<1,-\infty \leq \mathbf{E} \log \rho<0 .
$$

Observe, that the class of multi-type branching processes in random environment satisfying Assumptions $\mathrm{A} 0$ and A1 is an analog of the strongly subcritical case of single-type branching processes in random environment considered in (7). 
Set

$$
\begin{gathered}
\zeta:=\sum_{t \in \mathbf{N}_{0}^{p}} \sum_{i=1}^{p} v_{i} \sum_{j, k=1}^{p} \mathbf{F}^{(i)}(t) t_{j} t_{k}, \eta=\zeta /\left(\rho^{2} v^{*}\right), \\
\zeta_{n}:=\sum_{t \in \mathbf{N}_{0}^{p}} \sum_{i=1}^{p} v_{i} \sum_{j, k=1}^{p} \mathbf{F}_{n}^{(i)}(t) t_{j} t_{k}, \eta_{n}=\zeta /\left(\rho_{n}^{2} v^{*}\right),
\end{gathered}
$$

where $v=\left(v_{1}, \ldots, v_{p}\right)$ is from $4, v^{*}=\min \left(v_{1}, \ldots, v_{p}\right)$.

Introduce the random variables

$$
Q^{(i)}(n)=\mathbf{P}\left(Z(n) \neq \overrightarrow{0}^{T} \mid Z(0)=e_{i}, \Pi\right), Q(n)=\left(Q^{(1)}(n), \ldots, Q^{(p)}(n)\right)
$$

and let

$$
q_{i}(n)=\mathbf{P}\left(Z(n) \neq \overrightarrow{0}^{T} \mid Z(0)=e_{i}\right)=\mathbf{E} Q^{(i)}(n) .
$$

Note that under Assumptions $\mathrm{A} 0$ and $\mathrm{A} 1 Q^{(i)}(n) \rightarrow 0$ a.s. as $n \rightarrow \infty$ for all $1 \leq i \leq p$, since in view of (6)

$$
(v, Q(n)) \leq \min _{0 \leq k \leq n-1}\left|v M_{0} \cdots M_{k}\right| \leq \exp \left\{\min _{0 \leq k \leq n-1} S_{k}\right\} \rightarrow 0
$$

as $n \rightarrow \infty$. Denote by $u(n)=\left(u_{1}(n), \ldots, u_{p}(n)\right)^{T}:=u\left(M_{0} \cdots M_{n}\right), n \geq 0$, the right eigenvector of the product $M_{0} \cdots M_{n}$, corresponding to the Perron root $\rho\left(M_{0} \cdots M_{n}\right)=\rho_{0} \cdots \rho_{n}$.

The main result of the paper is the following statement.

Theorem 1 Suppose that Assumptions $A O$ and Al are valid, and

$$
\mathbf{E}\left(\rho \log ^{+} \zeta\right)<\infty
$$

where $\zeta$ is from (7). Then, as $n \rightarrow \infty$,

$$
q_{i}(n) \sim c_{i}(\mathbf{E} \rho)^{n}, c_{i}>0, i=1, \ldots, p .
$$

In conclusion of this section we give an example where condition 87 is fulfilled.

Since

$$
x \log ^{+}\left(y / x^{2}\right) \leq x \log ^{+} y+2 \sup _{t>0} t \log (1 / t) \leq x \log ^{+} y+2 / e, x>0, y>0,
$$

we see that if the measure $\mathbf{F}$ generating our random environment has a bounded support, i.e., there exists a $p$-dimensional cube

$$
K=\left\{t=\left(t_{1}, \ldots, t_{p}\right) \in \mathbf{N}_{0}^{p}, 0 \leq t_{i} \leq b, b>0,1 \leq i \leq p\right\},
$$

such that $\mathbf{P}(\mathbf{F}(K)=1)=1$, then condition 8 holds. 


\section{Auxiliary results}

The following assertion for the asymptotics of $u(n)$ has been obtained in (5).

Theorem 2 If Assumption $A 0$ is valid, then there exist a random vector $u=\left(u_{1}, \ldots, u_{p}\right)^{T}$ and a function $g(n) \geq 0, g(n) \rightarrow 0, n \rightarrow \infty$, such that with probability 1

$$
\left|u_{i}(n)-u_{i}\right| \leq g(n), i=1, \ldots, p .
$$

In addition,

$$
(v, u)=1, \alpha \leq u_{i} \leq 1 / v^{*},
$$

$v^{*}=\min \left(v_{1}, \ldots, v_{p}\right)$ and $v=\left(v_{1}, \ldots, v_{p}\right)$ is from (4).

The following theorem describes the behavior of $Q_{i}(n)$ as $n \rightarrow \infty$.

Theorem 3 Assume Assumption AO and let

$$
\mathbf{E} \log \rho<0
$$

Then with probabilty 1 , as $n \rightarrow \infty$,

$$
\frac{Q_{i}(n)}{(v, Q(n))} \rightarrow u_{i}, i=1, \ldots, p,
$$

where $u=\left(u_{1}, \ldots, u_{p}\right)^{T}$ is from Theorem 2

Note that relation (11) has been proved in (5) for the case when Assumption A0 and condition

$$
\mathbf{P}\left(S_{n}>0\right) \rightarrow a, n \rightarrow \infty,
$$

where $0<a<1$, are valid ( see Theorem 2 in (5)). The arguments presented in (5) are still valid if we replace condition $(12)$ by $(10)$.

We need the following notations. For $s \in J^{p}$ set

$$
\begin{gathered}
F_{k, n}(s)=F_{k}\left(F_{k+1}\left(\ldots F_{n-1}(s)\right)\right), 0 \leq k<n-1, F_{n, n}(s)=s, \\
F_{k, n}(s)=F_{k-1}\left(F_{k-2}\left(\ldots F_{n}(s)\right)\right), k>n \geq 0 .
\end{gathered}
$$

It is not difficult to see that $Q(n)=\overrightarrow{1}-F_{0, n}(\overrightarrow{0})$ and

$$
\begin{gathered}
\frac{1}{\left(v, \overrightarrow{1}-F_{0, n}(s)\right)}=\frac{e^{-S_{0}}}{\left(v, \overrightarrow{1}-F_{0, n}(s)\right)} \\
=\frac{e^{-S_{n}}}{\left(v, \overrightarrow{1}-F_{n, n}(s)\right)}+\sum_{k=0}^{n-1}\left(\frac{e^{-S_{k}}}{\left(v, \overrightarrow{1}-F_{k, n}(s)\right)}-\frac{e^{-S_{k+1}}}{\left(v, \overrightarrow{1}-F_{k+1, n}(s)\right)}\right) \\
=\frac{e^{-S_{n}}}{(v, \overrightarrow{1}-s)}+\sum_{k=0}^{n-1} e^{-S_{k}} g_{k}\left(F_{k+1, n}(s)\right), s \in J^{p},
\end{gathered}
$$

where

$$
g_{k}(s):=\frac{1}{\left(v, \overrightarrow{1}-F_{k}(s)\right)}-\frac{1}{\rho_{k}(v, \overrightarrow{1}-s)}, k \geq 0, s \in J^{p} .
$$

The following bound for $g_{n}(s), n \geq 0$, is from Lemma 1 in (5) 
Lemma 1 Assume Assumption A0. Then

$$
0 \leq g_{n}(s) \leq \eta_{n}, n \geq 0, s \in J^{p} .
$$

We need also the following statement.

Lemma 2 If Assumption $A 0$ and condition (10) are valid, then, as $n \rightarrow \infty$,

$$
\mathbf{E} Q^{(i)}(n) \sim \mathbf{E}\left(u_{i}(n)(v, Q(n))\right), i=1, \ldots, p .
$$

The proof of the lemma is elementary (it is based on Theorems 2, 3) and Fatou's lemma and we omit it.

\section{Proof of the main result}

We need some additional notation. Let $\Delta_{2}$ be the set of all possible tuples $f(s)=\left(f^{(1)}(s), \ldots, f^{(p)}(s)\right), s \in$ $J^{p}$, where $f^{(i)}(s), i=1, \ldots, p$, are $p$-dimensional probability generating functions.

In fact we identify the set $\Delta_{2}$ with the space $(\Delta, \mathcal{B}(\Delta))$. Since Assumption A1 yields $\mathbf{E} \rho<1$ we can introduce the random vector

$$
\bar{F}(s)=\left(\bar{F}^{(1)}(s), \ldots, \bar{F}^{(p)}(s)\right)^{T}, s \in J^{p},
$$

where $\bar{F}^{(i)}(s), i=1, \ldots, p$, are multidimensional (random) generating functions such that

$$
\mathbf{E} \varphi(\bar{F}(s))=\frac{\mathbf{E}(\rho \varphi(F(s))}{\mathbf{E} \rho}
$$

for every non-negative measurable function $\varphi$ on $\Delta_{2}$. ( Recall, that $F(s)$ was defined by (2)). In a similar way we introduce, for $n \geq 0$,

$$
\bar{F}_{n}(s)=\left(\bar{F}_{n}^{(1)}(s), \ldots, \bar{F}_{n}^{(p)}(s)\right)^{T}, s \in J^{p},
$$

by the relation

$$
\mathbf{E} \varphi\left(\bar{F}_{n}(s)\right)=\frac{\mathbf{E}\left(\rho \varphi\left(F_{n}(s)\right)\right)}{\mathbf{E} \rho},
$$

where $F_{n}(s)$ was defined by (3). It is not difficult to see that $\bar{F}_{0}(s), \ldots, \bar{F}_{n-1}(s)$ are i.i.d. copies of $\bar{F}(s)$ and

$$
\mathbf{E} \varphi\left(\bar{F}_{0}(s), \ldots, \bar{F}_{n-1}(s)\right)=\frac{\mathbf{E}\left(\exp \left(S_{n}\right) \varphi\left(F_{0}(s), \ldots, F_{n-1}(s)\right)\right)}{(\mathbf{E} \rho)^{n}},
$$

where $\varphi$ is from 15 .

Proof of Theorem 1. In view of Lemma 2 to prove Theorem 1 it is sufficient to show that

$$
\mathbf{E}\left(u_{i}(n)\left(v, \overrightarrow{1}-F_{0, n}(\overrightarrow{0})\right)\right) \sim c_{i}(\mathbf{E} \rho)^{n}, c_{i}>0, i=1, \ldots, p .
$$

Fix $i=1, \ldots, p$ and denote by

$$
u(n, 0)=\left(u_{1}(n, 0), \ldots, u_{p}(n, 0)\right)^{T}:=u\left(M_{n-1} M_{n-2} \cdots M_{0}\right), n \geq 1,
$$


the right eigenvector of the product $M_{n-1} M_{n-2} \cdots M_{0}$, corresponding to the Perron root $\rho\left(M_{n-1} M_{n-2}\right.$. $\left.\cdots M_{0}\right)=\rho_{n-1} \cdots \rho_{0}$. Using relation

$$
\mathbf{E}\left(u_{i}(n)\left(v, \overrightarrow{1}-F_{0, n}(\overrightarrow{0})\right)\right)=\mathbf{E}\left(u_{i}(n, 0)\left(v, \overrightarrow{1}-F_{n, 0}(\overrightarrow{0})\right)\right)
$$

and setting

in (15) we have

$$
\varphi\left(F_{0}(s), \ldots, F_{n-1}(s)\right)=\exp \left(-S_{n}\right)\left(v, \overrightarrow{1}-F_{n, 0}(s)\right)
$$

$$
\mathbf{E}\left(u_{i}(n)\left(v, \overrightarrow{1}-F_{0, n}(\overrightarrow{0})\right)\right)=(\mathbf{E} \rho)^{n} \mathbf{E}\left(\bar{u}_{i}(n, 0) \exp \left(-\bar{S}_{n}\right)\left(v, \overrightarrow{1}-\bar{F}_{n, 0}(\overrightarrow{0})\right)\right),
$$

where $\bar{u}_{i}(n, 0), \bar{S}_{n}, \bar{F}_{n, 0}$, etc. are the analogues of $u_{i}(n, 0), S_{n}, F_{n, 0}$, defined in the terms of the $\bar{F}_{i}, i \geq 0$. For instance, $\bar{S}_{n}=\sum_{i=0}^{n-1} \log \bar{\rho}_{i}$. Replacing $F_{k}$ by $\bar{F}_{k}$ in 13 we have

$$
\exp \left(-\bar{S}_{n}\right)\left(v, \overrightarrow{1}-\bar{F}_{n, 0}(\overrightarrow{0})\right)=\left(1+\sum_{k=1}^{n} \bar{\zeta}_{k-1} \exp \left(\bar{S}_{k}\right)\right)^{-1}
$$

where $\bar{\zeta}_{k}=\bar{g}_{k}\left(\bar{F}_{k, 0}(\overrightarrow{0})\right), k \geq 0$. Hence

$$
\mathbf{E}\left(u_{i}(n)\left(v, \overrightarrow{1}-F_{0, n}(\overrightarrow{0})\right)\right)=(\mathbf{E} \rho)^{n} \mathbf{E}\left(\bar{u}_{i}(n, 0)\left(1+\sum_{k=1}^{n} \bar{\zeta}_{k-1} \exp \left(\bar{S}_{k}\right)\right)^{-1}\right) .
$$

Observe that $\lim _{n \rightarrow \infty} \bar{u}_{i}(n, 0)=\lim _{n \rightarrow \infty} u_{i}(n)=u_{i}$ a.s. by Theorem 2 Thus, to prove 9 9 it suffices to show that with probability 1

$$
\sum_{k=1}^{\infty} \bar{\zeta}_{k-1} \exp \left(\bar{S}_{k}\right)<\infty
$$

By Lemma 1

$$
\bar{\zeta}_{k} \leq \bar{\eta}_{k}:=\sum_{t \in \mathbf{N}_{0}^{p}} \sum_{i=1}^{p} v_{i} \sum_{j, l=1}^{p} \overline{\mathbf{F}}_{k}^{(i)}(t) t_{j} t_{l} /\left(\bar{\rho}_{k}^{2} v^{*}\right), k \geq 0 .
$$

Condition 8 implies, that $\bar{S}_{n}, n \geq 0$, is a random walk with negative drift

$$
\mathbf{E} \log \bar{\rho}=\frac{\mathbf{E}(\rho \log \rho)}{\mathbf{E} \rho}<0 .
$$

Since by the strong law of large numbers $\lim _{n \rightarrow \infty} \bar{S}_{n} / n=\mathbf{E} \log \bar{\rho}$ a.s., to prove 16 it suffices to show that

$$
\lim _{n \rightarrow \infty} \sup \frac{\log ^{+} \bar{\eta}_{n}}{n}=0 \text { a.s. }
$$

Using [15, (8), and the estimate $\sup _{x>0}(x \log 1 / x)=e^{-1}$, it is not difficult to check that

$$
\begin{gathered}
\mathbf{E} \log ^{+} \bar{\eta}_{n}=\mathbf{E} \log ^{+} \frac{\bar{\zeta}_{n}}{\bar{\rho}_{n}^{2}} \leq \frac{1}{\mathbf{E} \rho}+\mathbf{E} \log ^{+} \bar{\zeta}_{n} \\
=\frac{1}{\mathbf{E} \rho}+\frac{\mathbf{E}\left(\rho \log ^{+} \bar{\zeta}\right)}{\mathbf{E} \rho}<\infty .
\end{gathered}
$$

Relation (17) follows from 18. This entails the desired result. 


\section{References}

[1] V.I. Afanasyev, J. Geiger, G. Kersting, and V.A. Vatutin, Criticality for branching processes in random environment. Ann. Prob. (2005) 33, N 2, 645-673.

[2] K.B. Athreya and P.E. Ney, Branching Processes. Springer, Berlin, 1972.

[3] K.B. Athreya and S. Karlin, On branching processes with random environments: I,II. Ann. Math. Statist (1971) 42, N 5, 1499-1520; (1971) 42, N 6, 1843-1858.

[4] E.E. Dyakonova, J. Geiger and V.A. Vatutin, On the survival probability and a functional limit theorem for branching processes in random environment. Markov Process. Related Fields (2004) 10, N 2, 289-306.

[5] E.E. Dyakonova, Critical multitype branching process in random environment. Discrete Math. Appl. (2007) 17, N 6, 587-606.

[6] J. Geiger and G. Kersting, The survival probability of a critical branching process in random environment. Theory Probab. Appl. (2000) 45, N 3, 607-615.

[7] J. Geiger, G. Kersting, and V.A. Vatutin, Limit theorems for subcritical branching processes in random environment. Ann. I.H. Poicare (2003) 39, N 4, 593-620.

[8] N. Kaplan, Some results about multidimentional branching processes with random environments. Ann. Prob. (1974) 2, N 3, 441-455.

[9] M.V. Kozlov, On the asymptotic behavior of the probability of non-extinction for critical branching processes in a random environment. Theory Probab. Appl. (1976) 21, N 4, 791-804.

[10] B.A. Sevastyanov Verzweigungprozesse. Akademie, Berlin, 1974.

[11] W.L. Smith and W. Wilkinson, On branching processes in random environment. Ann. Math. Statist (1969) 40, N 3, 814-827.

[12] D. Tanny, On multitype branching processes in a random environment. Adv. Appl. Prob. (1981) 13, N 3, 464-497.

[13] V.A. Vatutin and E.E. Dyakonova, Galton-Watson branching processes in random environments, I: limit theorems. Theory Probab. Appl. (2003) 48,N 2, 314-336.

[14] V.A. Vatutin and E.E. Dyakonova, Branching processes in random environment and bottlenecks in the evolution of populations. Theory Probab. Appl. (2006) 51 , N 1, 189-210.

[15] E. W. Weissener, Multitype branching processes in random environments. J. Appl. Prob. (1971) 8, N 1, 17-31. 